Sección dos: Textos

Educación y Exclusión social

\title{
Educación y movilidad de la comunidad rom en Madrid: el caso del gallinero ${ }^{1}$
}

Education and mobility of the rom community in Madrid: the case of el Gallinero

José David Gutiérrez Sánchez

jotadegs@hotmail.com

Universidad Pablo Olavide

Jenifer Ortega Torres

jenniferortegatorres@gmail.com

\section{Resumen}

El trabajo que nos ocupa analiza la situación en la que viven las familias gitanas y de procedencia rumana en el asentamiento chabolista de El Gallinero, teniendo en cuenta la movilidad del colectivo por Europa y además como los agentes sociales intervinientes afrontan la práctica educativa como propuesta y práctica inclusiva para prevenir y combatir el absentismo escolar en contextos catalogados como de riesgo. El análisis de las prácticas comunitarias y los actores que intervienen nos ha permitido identificar aquellas que son positivas para el fomento de la convivencia por medio de la participación social.

Palabras clave: educación, movilidad, infancia, rumanos, inclusión social.

\begin{abstract}
The present work analyzes the situation in which the Romanian families of Romanian origin live in the shanty town of El Gallinero, taking into account the mobility of the group in Europe and also as the intervening social agents face the educational practice as a proposal and Inclusive practice to prevent and combat school absenteeism in contexts classified as risk. The analysis of community practices and the actors involved has allowed us to identify those that are positive for the promotion of coexistence through social participation.
\end{abstract}

${ }^{1}$ Recibido: 09/05/ 2018 Evaluado: 15/06/2018 Aceptado: 20/07/2018 
Keywords: education, mobility, childhood, Romanians, social inclusion.

\section{Introducción}

En los últimos años ha suscitado ser de gran interés para las Ciencias Sociales y de la Educación el papel que desempeñan determinados grupos, con gran carga de exclusión social, en cuanto a las estrategias de desarrollo que emplean en contextos denominados como de riesgo. Desde 2010, con la colaboración de investigadores y profesionales de la intervención comunitaria en el asentamiento chabolista de El Gallinero, realizamos un análisis de la situación en el que una comunidad de etnia gitana y procedencia rumana habita en condiciones de extrema vulnerabilidad (Gutiérrez, 2017). El Gallinero, cuya denominación le proviene por la ubicación en una antigua granja avícola, data aproximadamente desde 2001 fruto del derribo de las Unidades de Realojamiento Especial de la Quinta, La Celsa, La Rosita y, sobre todo, de las actuaciones desarrolladas para desmantelar el Poblado de las Barranquillas propiciado por el Ayuntamiento de Madrid a finales de los años noventa.

El Gallinero se ha configurado como un asentamiento de aproximadamente unas 400 personas de las que el $50 \%$ son menores de edad. Se trata de un escenario situado a 14 kilómetros de la ciudad de Madrid y a un kilómetro de la Cañada Real Galiana. Según Monreal (2014), el desuso de la Cañada como vía pecuaria y su proximidad a la ciudad de Madrid, pueden argumentar la ocupación de estos terrenos desde 1960, los cuales vieron incrementada su ocupación desde los años 90, donde localizamos a diversos grupos de población como el colectivo gitano rumano, siendo éste el objeto de este estudio. En contraste, existe un gran número de proyectos públicos y privados que tratan de mejorar la situación de los sujetos, convirtiendo a El Gallinero en un objetivo "prioritario" de las actuales políticas pero, sin embargo, dichas ayudas "tropiezan" con los continuos intentos de desalojos y desahucios que se llevan a cabo desde las administraciones (local y regional) en un intento de desocupar unos terrenos que son de titularidad privada.

La elección del asentamiento a estudiar, en este caso, buscaba una experiencia dilatada en el tiempo en intervención comunitaria con una población que emplea la movilidad por Europa como estrategia para mejorar su calidad de vida. Se trata, además, de analizar cómo contextos de riesgo como El Gallinero, desarrollan iniciativas educativas para la infancia y, además, cómo la movilidad constante y los problemas propios por vivir en entornos inapropiados para el hábitat, inciden en la educación y como ésta se mantiene con el tiempo.

La investigación identifica dos claves para interpretar la fractura social que se observa en este trabajo (Epstein y Kiszbaum, 2016). De un lado, este tipo de barrios o asentamientos son escenarios comunitarios con gran carga cultural como otros existentes en Europa que, como bien señala Gimeno (2017), oscilan entre la coexistencia y la hostilidad, donde es posible detectar un clima de desbordamiento y estancamiento entre los profesionales de lo social lo cual se relaciona, a su vez, con carencias en sus competencias interculturales (Leiva, 2017) y formación en el enfoque comunitario (Gutiérrez, 2017; Marchioni, 2007). Y por otro lado, es oportuno aproximarse a las estrategias de deserción de los vecinos (Gimeno, 2017), que huyen de la intervención (en determinados momentos) técnica y política, lo cual termina desorientando a los profesionales que operan en el lugar (Virno, 2003; Sorando, 2004). 


\section{Marco Teórico}

Con la adhesión de diez países del Centro y Este de Europa (Chipre, República Checa, Estonia, Hungría, Letonia, Lituania, Malta, Polonia, Eslovaquia y Eslovenia) en el año 2004, alrededor de 1,5 millones de personas pertenecientes a la minoría étnica gitana empezaron a ser, formalmente, ciudadanos de la Unión Europea (en adelante UE). Con la siguiente ampliación, en el año 2007 y, la adhesión de Rumanía y Bulgaria, el número de personas pertenecientes a dicha minoría en la UE se incrementó hasta los 4,4 millones (Petrova, 2004).

Rumanía se caracterizó por poseer uno de los sistemas más cerrados y dictatoriales del Este de Europa a partir de la Segunda Guerra Mundial, durante más de cuatro décadas (19441989). A partir del año 1989, con la caída del régimen de Ceaucescu, Rumanía inició una transición hacia un nuevo modelo político y económico, sin embargo, este proceso al igual que en otros países del Este, fue bastante complejo, teniendo un importante impacto en las condiciones de vida de la población (Macías, 2005).

En el año 1993, en la Cumbre de Copenhague, se establecieron los requisitos que debían cumplir los países candidatos a formar parte de la Unión Europea. Estos requisitos se resumían en los siguientes criterios:

1. Criterios políticos: instituciones que garanticen la democracia, la ley, los derechos humanos y la protección de las minorías.

2. Criterios económicos: los estados miembros deben alcanzar el estatus de FME (Funcionating Market Economy), para lo que resultará necesario la liberalización de los precios y el mercado, el fortalecimiento de un sistema legal y los derechos de la propiedad. Además, también se incluyen la estabilidad macroeconómica, un adecuado sector financiero y la ausencia de barreras para la entrada y salida del mercado.

3. Adopción del Acerbo Comunitario (Acquis communautaire): es decir, el conjunto de derechos y obligaciones comunes a todos los estados miembros de la Unión Europea.

A partir del año 1989, los sucesivos gobiernos implementaron una serie de medidas económicas y políticas en Rumanía con la intención de transformar la economía interior con el objetivo de alcanzar la denominada FME, procediendo a la privatización de empresas estatales y al cierre de otras industrias obsoletas, produciéndose así despidos masivos y aumentando, considerablemente, los niveles de desempleo. Asimismo, a partir del año 2007, han tenido lugar transformaciones importantes en las esferas económica, política y social para alcanzar los estándares europeos establecidos. El proceso de transición resultó en una crisis económica sin precedentes que incidió en un incremento importante de los niveles de pobreza, siendo uno de los colectivos más perjudicados el de etnia gitana (Gutiérrez, 2017), el cual constituía hogares numerosos, baja formación y, además, vivían en el hábitat rural, mermando seriamente las condiciones de vida. 
Son éstas, entre otras razones, las que se encuentran en la explicación del fenómeno itinerante de los gitanos rumanos. Siguiendo a Crowe (2003), los movimientos de esta población podrían considerarse una estrategia de supervivencia que se desarrolla bajo las distintas circunstancias vividas a lo largo del tiempo.

La movilidad de los gitanos rumanos no es un nuevo fenómeno migratorio en sí. Las distintas políticas de los gobiernos y tratamiento que han recibido en los distintos momentos históricos y regiones han incidido en los patrones de movimiento de esta población. Además, y con la incorporación de Rumanía a la UE, ya no es apropiado referirnos al colectivo como migrantes, ya que se convierten desde 2007 en ciudadanos europeos.

En Rumanía, los gitanos fueron asociados automáticamente con las difíciles situaciones que atravesaba el país. Muchos de ellos viajaron a países como Alemania, Austria o Francia sin embargo, los obstáculos a la inmigración impuestos por estos países hizo que la movilidad se orientara hacia los países de la cuenca mediterránea: España, Italia o Portugal (Marcu, 2013).

Tal y como hace referencia Marcu (2013), en la emigración rumana hacia España, los investigadores señalan tres etapas diferenciadas. La primera de ellas, comprendida entre 1990-1995, cuando se registraron 325.999 solicitudes de asilo político en Europa Occidental (Diminescu, 2003). Según las estadísticas del año 2006, entre 1990 y 1995, España atrajo un número muy reducido de rumanos sin embargo, a partir de 1996, las rutas internacionales cambiaron de dirección, dirigiéndose hacia países del sur, siendo Italia y España los principales destinos. El periodo 2002-2007, estuvo marcado por la apertura fronteriza mediante el Tratado de Schengen que supuso la libre circulación de personas en el territorio comunitario. Esta etapa se configura como una de las más importantes en la emigración rumana hacia España. El último periodo comienza en el año 2007 y está marcado por el ingreso de Rumanía en la UE. En solo un año, los flujos aumentaron desde 211.325 a 603.889, convirtiéndose los rumanos en ciudadanos europeos con libre derecho de circulación (Favell, 2008).

Por su parte, la distribución geográfica en territorio español se relaciona con la presencia de familiares y amigos en las principales ciudades y provincias de destino y, por supuesto, con la oferta de empleo, mayor en las áreas urbanas y litorales. Destaca la concentración de efectivos en Madrid (30\%) y el litoral mediterráneo entre Girona y Almería (34\%), sobre todo en la provincia de Castellón (Viruela, 2006). No obstante, se observa un intenso proceso de dispersión por buena parte del territorio, en particular por las provincias próximas a Madrid y por el valle del Ebro, debido al rápido aumento del stock de ciudadanos rumanos y a la movilidad geográfica en una constante búsqueda de oportunidades (Viruela, 2006).

Los gitanos rumanos se han constituido como el grupo más numeroso de la etnia gitana procedente de los países del Este de Europa en España. Gitanos búlgaros y unos pequeños grupos de gitanos serbios, checos o Macedonios completan el resto de procedencias (López Bustamante, 2005). 
El nivel educativo, es otro de los que se ha visto fuertemente afectado por las transformaciones políticas, sociales y económicas que han tenido lugar en Rumanía. Siguiendo a López Bustamante (2005), la mayor parte de los menores gitanos en Rumanía son discriminados en el derecho a una educación digna, a pesar de estar éste garantizado por ley. Este aspecto, el cual ya parte desde origen, se puede mantener todavía en el país destino, es decir, la carga de discriminación que sufren los niños y niñas se muestra compleja en cuanto su abordaje a lo largo del tiempo. Según Leiva (2017), esta perspectiva, en la que se etiqueta al alumno inmigrante como "culturalmente desvalido", tiene un resultado nítido y claro en términos de negacionismo de éxito y déficit en la construcción identitaria de los jóvenes procedentes de minorías étnicas (Harris, 2004).

Siguiendo a Giménez (2011, p.57):

La educación es tanto un derecho fundamental como un instrumento necesario para que todas las personas puedan desarrollar sus capacidades y acceder a un nivel de vida adecuado, especialmente, los grupos más vulnerables que encuentren en ella una de las pocas vías para salir de su situación de exclusión social.

En los últimos años, la cuestión sobre el éxito y el fracaso escolar de los estudiantes en los distintos sistemas educativos se ha convertido en un tema prioritario y uno de los mayores desafíos de los países de todo el mundo (Leiva, 2017). El fracaso escolar como fenómeno educativo es todavía aún más grave en contextos de riesgo y vulnerabilidad (Gutiérrez, 2017). Es por tanto pertinente incidir en aquellos aspectos culturales y sociales que se observan dentro de la problemática.

Por otro lado Aguaded y Maraver (2014) reflejan como la infancia es una etapa vital de importancia para el desarrollo humano, por lo que en función de cómo se aborde dicha cuestión, es posible prevenir posibles situaciones de riesgo. Dentro de la investigación cualitativa el concepto de riesgo se sitúa como complejo y multifacético (Warner, 1992; Ryan, 1996; Stevenson, 1999; Gutiérrez y Diz, 2017). En nuestro caso entendemos el riesgo como probabilidad de que se desarrollen determinadas situaciones (Gutiérrez, 2014).

\section{Metodología}

El presente artículo expone los resultados de un proceso de investigación mediante la aplicación de técnicas y métodos cualitativos en el asentamiento chabolista de El Gallinero entre 2010-2017. El objetivo central de este trabajo era: "realizar una investigación evaluativa de la intervención social con menores gitanos rumanos con el fin de establecer estrategias para proyectos de Trabajo Social con dichos colectivos" (Gutiérrez, 2017, p.76). El caso que nos ocupa y, más concretamente, en este artículo, nos detenemos en los diversos aspectos educativos que se han dado lugar en dicho lugar y que soportan alteraciones por la movilidad constante del colectivo.

La metodología empleada contaba en primer lugar con un análisis documental (planes, proyectos, memorias, evaluaciones, etc.) de las iniciativas, tanto públicas como privadas, orientadas a la población asentada en El Gallinero. Para comprender las intervenciones que se desarrollaban en el asentamiento, hemos analizado los proyectos de intervención social 
orientados tanto al ámbito educativo formal como no formal partiendo de la incorporación en las actividades que la Fundación Imaginario Social llevabas a cabo por medio del Proyecto Nido durante 2010-2014.

Además, también hemos realizado entrevistas (un total de 86) entre niños y niñas, familiares, técnicos de proyectos y líderes locales. El propósito de las entrevistas en este estudio fue que cada entrevistado proporcionara una información específica de las intervenciones sociales en las que ellos participaban y además, en el caso de los menores, analizar qué aspectos afectan al desarrollo de los mismos y conocer las causas del porqué determinados proyectos no terminan estableciéndose en el asentamiento (Gutiérrez, 2017a).

También hemos complementado este trabajo de campo con la observación participante en el propio asentamiento y en aquellas actividades de carácter comunitario, así como con el empleo de técnicas de investigación acción-participativas (IAP). La IAP se llevó a cabo como medio para poder analizar la realidad social de la comunidad asentada e indagar sobre aquellos procesos participativos sociales dentro del grupo de jóvenes y la comunidad (Valderrama, 2013).

El Proyecto Nido tenía como objetivo central la intervención socioeducativa con jóvenes menores de edad por medio de actividades de educación no formal. Según Fals-Borda (1985) en relación a la IAP, la inserción del investigador en la comunidad tiene el objetivo de analizar las condiciones históricas y estructura social, estudiando el nivel de desarrollo de conciencia de los miembros de una determinada comunidad sobre los problemas focalizados, el desarrollo de las organizaciones políticas y grupos de acción y el compromiso a la solución de problemas con la comunidad o grupo (Balcazar, 2003). Según Selener (1997) el grado de participación de los miembros de la comunidad o grupo determina el nivel de IAP.

\section{Resultados}

A lo largo de la investigación se han obtenido diversos resultados en torno a la educación dentro del colectivo gitano rumano asentado en El Gallinero. Para sintetizar mejor la información vamos a categorizar los resultados en los siguientes puntos:

\section{Educación y Etapas de desarrollo}

Durante la investigación se han observado dos etapas diferenciadas que inciden directamente en la educación formal y no formal de los niños y niñas. La primera de ellas que denominamos como "Etapa Familiar-escolar", se extiende desde los 0 a los 13 años, donde el menor todavía se interesa por el aprendizaje y en la que los padres, generalmente, no suelen impedir que sus hijos estudien. Y, la segunda "Etapa de proyección a la vida adulta", a partir de los 13 años, especialmente significativa en las niñas, ya que es a partir de esta edad cuando las menores comienzan a contraer matrimonio (Gutiérrez, 2015).

Estas etapas son notables por el papel que la educación formal y no formal inciden sobre ellas. En la primera de ellas no existe una influencia negativa del entorno por abandonar los estudios. En esta etapa los profesionales de la intervención social por medio de programas y 
proyectos educativos (como es el Proyecto Nido) tratan de incidir en la relación de los padres con los menores, de tal forma que esta etapa persista el máximo tiempo posible, lo que se traduce en reducir las tasas de analfabetismo y absentismo escolar. Se tiene en cuenta que la familia es una complejidad organizada compuesta de subsistemas en mutua interacción (Mondragón y Trigueros, 2004), lo cual si algunos de dichos subsistemas "falla" en su funcionamiento, la totalidad del sistema se ve afectado. Con ello se acentúa el trabajo que tiene que constituirse entre profesionales de la intervención, familias y los sujetos de la acción, mostrando la necesidad de salvaguardar el periodo el máximo tiempo posible, con una buena estructura y división de tareas. En esta etapa no se aprecian diferencias considerables entré género, ya que en la mayoría de los casos, se constata la concepción de que el menor tiene todavía que desarrollarse.

La segunda etapa (Proyección a la vida adulta), los niños y niñas van asumiendo nuevos roles y empiezan a colaborar con mayor intensidad en tareas del hogar e incluso a trabajar en empleos eventuales relacionados con la economía sumergida y la mendicidad. La influencia del entorno es característico de este estadío, ya que la percepción de la comunidad de El Gallinero en cuanto que los jóvenes a una determinada edad tienen que dejar de ser niños para convertirse en hombres y mujeres, acentúa aún más el problema que deriva de este ciclo, provocando efectos como, abandono de la escuela, aumento del analfabetismo, asimilación de actividades propias de la delincuencia (robos y hurtos) y un cambio de actitud drástico que refleja la comprensión por parte de los jóvenes de sus circunstancias dentro de la comunidad donde viven y se relacionan (Gutiérrez, 2015).

La segunda etapa, también coincide con el comienzo del casamiento, la cual es mayormente cometida por las chicas. Este aspecto afecta, a su vez, a la posibilidad de continuar con los estudios y poder enrolarse en actividades extraescolares. En este sentido, sería conveniente re-plantearse la formación del profesorado en materia de interculturalidad (Leiva, 2012), ya que ayudaría a que por lo menos el nivel de estudios cursados fuese más alto y, a su vez, a reducir la probabilidad de comenzar tempranamente a trabajar, casamiento u ocuparse de tareas del hogar.

\section{Movilidad}

Como reflejábamos anteriormente, hablamos de un sector de la población europea que lleva a cabo una movilidad constante entre países (como por ejemplo Francia, Italia, Portugal y España). Este aspecto que, no siendo considerado como negativo, sí afecta indirectamente a la educación de los niños y niñas. Se trata de una movilidad de carácter familiar que tiene mayoritariamente un motivo económico (Pajares, 2007). Por lo tanto, la movilidad del colectivo entorpece, en determinadas situaciones, la continuidad de la asistencia a la escuela y, a su vez, las relaciones con otros alumnos y profesores. Este factor incide en diversas situaciones negativamente ya que, no permite que los niños y niñas sigan relacionándose (con lo positivo que puede resultar para la inclusión social) y, además, poder aprender una lengua nueva correctamente.

Entidades sociales no lucrativas 
En este punto resulta necesario destacar el papel relevante que las entidades sociales llevan a cabo en el asentamiento en relación a la educación. Proyectos como el de la Fundación Imaginario Social (Proyecto Nido) u otros como Henry Dunant (Cruz Roja), han destacado por ser intervenciones con gran éxito durante el periodo entre 2010-2014. Ambos proyectos tenían la misión de reducir los niveles de absentismo escolar y, además, concienciar a los padres y madres de la importancia de la escolarización.

Es trascendente señalar cómo a edades superiores, a partir de 13 años, era más complejo el trabajo con los chicos y chicas por las cuestiones de movilidad y exigencias comunitarias. En este punto señalamos la importancia que tiene incorporar planes de formación profesional, orientados a la búsqueda de empleo y ayudas en entornos familiares económicamente bajos.

Un aspecto destacado en este punto resultó ser la capacidad de financiación de las propias entidades, es decir, en un contexto de crisis económica como el que vive Europa y sobre todo los países del Sur, las entidades sociales ven recortados sus presupuestos por lo que a veces puede afectar directamente a la capacidad de incidencia por medio de proyectos o planes en un territorio concreto. Se observó que a mayor capacidad o seguridad económica la posibilidad de cumplir con los objetivos aumenta considerablemente por lo que ante los retos que supone la educación en contextos como de riesgo o vulnerabilidad como el que nos ocupa, es de interés mantener la financiación acorde con los objetivos previamente propuestos.

\section{Actividades socioeducativas en los proyectos de intervención}

Cabe destacar, dentro de la investigación, cómo determinadas actividades de corte socioeducativo propiciaban efectos positivos dentro del interés por mejorar la situación de exclusión social y educativa $\mathrm{y}$, además, convertir a los agentes sociales en canales permanentes de ayuda entre los colegios e institutos y el asentamiento. Actividades de apoyo escolar, desarrollo de habilidades sociales, deportivas, salidas y excursiones puntuales a teatros, cines o campamentos permitían una mejor relación entre los sujetos y los agentes de la intervención. Además, la creación de "contratos sociales" donde por un lado el niño o niña se comprometía a ir a la escuela y aprobar las materias y, por otro, las entidades conseguían financiación para visitar lugares de distensión, resultaba ser una buena praxis y reflejaba mejoras en el comportamiento. Por supuesto, esto no siempre se reproducía con éxito por parte de los jóvenes, pero se hacían sin embargo grandes avances y fomentaba más la relación entre las familias y las entidades sociales.

\section{Agentes Externos}

Otro de los aspectos que inciden son aquellos relacionados con los desalojos y redadas que se llevan a cabo en el asentamiento. Las redadas (debido sobre todo por la relación de los sujetos con el robo de cobre) incidían negativamente en la población ya que, durante la realización de actividades, éstas causaban que se cortara con la dinámica de las mismas, con la movilización social de todo el asentamiento. La cantidad de redadas llevadas a cabo durante la noche afectaban a la conciliación del sueño en los niños y niñas, con la posterior falta al día siguiente de acudir a la escuela, debido al miedo y el cansancio. Otros factores externos detectados son: incendios en chabolas, inundaciones por irregularidades en el 
terreno, proximidad con vertederos, plagas de roedores e insectos, las cuales afectaban notablemente a la realización de actividades.

En relación a los desalojos hay que decir que, debido al asentamiento en un terreno de titularidad privada, el Ayuntamiento de Madrid durante 2010-2014 llevó a cabo numerosos intentos de desalojos como táctica de presión para que la población de alguna forma desocupara el lugar. A la vez que con las redadas, éstos hechos producían mucho estrés a la población lo que afectaba a la concentración y además, debido a la complejidad del problema, los niños y niñas debían permanecer con sus padres cortando nuevamente la dinámica educativa.

\section{Discusión y Conclusiones}

En el discurso en torno a las cuestiones educativas, día a día, se van construyendo y/o rescatando diversas nociones que resultan fundamentales al intentar explicar la situación de la educación y sus determinantes. Entre las nociones que aparecen continuamente en el debate se encuentran las condiciones sociales, económicas y políticas y, dentro de éstas, el fenómeno de la exclusión aparece inevitablemente. En este trabajo se observa la exclusión educativa, dentro de la cual nos referimos concretamente por un lado, al analfabetismo y la carencia de estudios, si abordamos la población en general de El Gallinero; y por otro, al abandono y las dificultades de acceso y mantenimiento en el sistema educativo reglado si nos referimos a menores y jóvenes.

La división por etapas del desarrollo de los niños, niñas y jóvenes que viven en el asentamiento chabolista de El Gallinero, nos ayudan a identificar los factores que se han de tener en cuenta para, de este modo, lograr una mejor intervención social, comprender las características de entornos o contextos de riesgo, permitir mayor entendimiento de la cultura, analizar el proyecto de movilidad de los gitanos rumanos en Europa, y por supuesto, establecer las mejores vías posibles para desarrollar una educación tanto formal como no formal teniendo en cuenta los factores externos que inciden en dicho territorio.

Es oportuno, desde el punto de vista de la intervención social, tener en cuenta qué aspectos inciden negativamente en la educación, es decir, es preciso como profesionales conocer con anterioridad al trabajo en dichos contextos de riesgo, las características propias de la población y el asentamiento como modo de poder resolver y solventar los problemas que pudieran establecerse debido a los altos niveles de analfabetismo y absentismo escolar. A pesar de dichos altos niveles, existen proyectos que han mejorado la situación, aunque todavía queda mucho por hacer, sobre todo a edades a partir de los 13 años como identificábamos en nuestra investigación.

Por último habría que abordar el papel del Estado en relación a qué medidas son necesarias adoptar en la intervención con familias de gran movilidad. Es importante conocer qué planes se han puesto en marcha para afrontar el establecimiento de las familias en entornos desfavorecidos y, además, cómo dichos espacios afectan a los niños y niñas en cuanto a su posición en la sociedad como sujetos a proteger, cómo adaptar la educación formal a una población con características propias y cómo incidir sobre los casamientos prematuros los cuales afectan, naturalmente, al desarrollo social y educativo. 


\section{Referencias}

Aguaded, J.I. \& Maraver, P. (2014). Infancia y medios de comunicación: de los riesgos a la apropiación creativa. En Jiménez, A.S., Pantoja, A., Leiva, J.J. y Moreno, E. (Coord.). Infancia en Contextos de Riesgo. XXV Años de la Convención sobre los Derechos del Niño. (pp. 203-209). Granada: GEU Editorial.

Balcazar, F.E. (2003). Investigación acción-participativa (IAP): Aspectos conceptuales y dificultades de implementación. Revista Fundamentos en Humanidades. Año IV-NI/ II (7/8),591-77.

Bustamante, J. L. (2005). Las pateras del asfalto: algunas consideraciones sobre la inmigración de los gitanos rumanos. I Tchatchipen: lil ada trin tchona rodipen romani-revista trimestral de investigación gitana, 51, 16-26.

Crowe, D. (2003). The International and Historial Dimensions of Gitanosni Migra- tion in Central and Eastern Europe. NATIONALITIES PAPERS 31(1): 81-94.

Diminescu, D. (2003). Introducción. En D. Diminescu (Ed.), Visibles Mais Peu Nombreux. Les Circulations Migratoires Roumaines, (pp. 1-23). Paris: Editions de la Maison des sciences de l'homme.

Epstein, R. y Kirszbaum, T. (2016). Territoires perdus versus territoires oubliés: les deux discours de la fracture. Urbanisme, 399, 57-59.

Fals Borda, O. (1985). Conocimiento y poder popular. Bogotá: Siglo XXI.

Favell, A. 2008. "The new face of East-West migration in Europe”. Journal of Ethnic and Migration Studies (34),701-716.

Giménez, S. (2011). Igualdad y no discriminación en el ámbito educativo. Informe anual Fundación Secretariado Gitano 2011. Discriminación y Comunidad Gitana. Madrid: FSG.

Gimeno, CH. (2017). Retos transfronterizos de la intervención en barrios multiculturales. Revista Andaluza de Ciencias Sociales, Anduli, (16), 53-72.

Gutiérrez, J.D. (2017). Movilidad del pueblo gitano de Europa del Este en el asentamiento chabolista de El Gallinero. Revista Andaluza de Ciencias Sociales, Anduli, (16), 73 89.

Gutiérrez, J.D. (2017a). La intervención social con menores inmigrantes rumanos: Proyectos comunitarios en "El Gallinero" (Madrid) 2010-2014 (Tesis Doctoral). Universidad de Sevilla, Sevilla. 
Gutiérrez, J.D. (2015). Los menores gitanos rumanos de "El Gallinero": Etapas de desarrollo en un contexto de riesgo. Revista Electrónica de Investigación y Docencia, REID, (13), 27-44.

Gutiérrez, J.D. (2014). Proyectos sociales en contextos de riesgo. En A.S Jiménez. (coord), Infancia, Cultura y Emoción. Un escenario internacional de formación (pp. 75-85). Granada: GEU Editorial.

Gutiérrez, J. D. y Diz, J. (2017). Cooperación Internacional en Marruecos: empoderamiento para insertar socio-laboralmente a jóvenes en contextos de riesgo. Cuadernos de Trabajo Social. 30 (1),163-174.

Harris, J. et al. (2004). African Americans and Multicultural Education. A Proposed Remedy for Disproportionate Special Education Placement and Underinclusion in Gifted Education. Education and Urban Society, 36 (3), 304-341.

Leiva, J.J. (2017). La interculturalidad como respuesta preventiva del fracaso escolar en contextos de riesgo. Revista Andaluza de Ciencias Sociales, Anduli, (16), 19-33.

Leiva, J.J. (2012). Aportaciones y reflexiones pedagógicas sobre educación intercultural: de la diversidad cultural a la cultura de la diversidad. Revista Qurriculum, (25), 57-75.

Macías, A. (2005). La población romaní en el flujo migratorio del Este hacia Europa occidental: el caso de Rumania. Documentación Social, (137), 79-98.

Marchioni, M. (2007). Planificación social y organización de la comunidad. Madrid: Editorial Popular.

Marcu, S. (2013). La movilidad transfronteriza de rumanos en España en tiempos de crisis. Revista Internacional de Sociología, 71 (1), 115-141.

Martínez, R. V. (2007). Migración y nuevas tecnologías de la información y la comunicación: inmigrantes rumanos en España. Migraciones. Publicación del Instituto Universitario de Estudios sobre Migraciones, (21), 259-290.

Mondragón, J. y Trigueros, I. (2004). Intervención con menores. Acción socioeducativa. Madrid: Narcea.

Monreal, P. 2014. Imágenes y representaciones de un espacio urbano: el papel de los medios de comunicación en la reproducción de las desigualdades. Antropológica, (33), 3966.

Pajares, M. (2007). Inmigrantes del Este. Procesos migratorios de los rumanos. Barcelona: Icaria.

Petrova, D. (2004). The Roma: Between a Myth and the future. Roma Rights Quaterly 
Proyecto Nido (2011). Proyecto Nido: Todos los niños/as tienen a una educación que asegure su integración social. Madrid: Fundación Imaginario Social.

Ruiz, A. P. (2006). Educación e inmigración en España. Revista española de educación comparada, (12), 339-364.

Ryan, T. (1996). Risk Management and People with Mental Health Problems. En H. Kemshall \& J. Pritchard (eds.), Good Practice in Risk Assessment and Risk Management, 1. Londres: Jessica Kigsley.

Selener, D. (1997). Participatory action research and social change. New York: Cornel University Participatory Action Research Network.

Sorando, D. (2014). Espacios en conflicto. Un análisis relacional del cambio social en los centros estigmatizados (Tesis Doctoral). Madrid: UCM.

Stevenson, O. (1999). Old People at Risk. Social Work, 36. Londres: Jessica Kingsley.

Virno, P. (2003). Virtuosismo y revolución. Madrid: Traficantes de Sueños.

Viruela, R. (2006). Inmigrantes rumanos en España. Aspectos territoriales y procesos de sustitución laboral. Scripta Nova. Revista Electrónica de Geografía y Ciencias Sociales. Vol. X, (222). Recuperado de http://cort.as/-8OPT

Warner, F. (1992). Introduction. Risk: Analysis, Perception and Management Report of a Royal Society Study Group. Londres: Royal Society. 